\title{
UKA closely preserves natural knee kinematics in vitro.
}

\author{
Thomas J. Heyse ${ }^{1}$ \\ Bilal F. El-Zayat ${ }^{1}$ \\ Ronny De Corte ${ }^{2}$ \\ Yan Chevalier $^{2}$ \\ Lennart Scheys ${ }^{2}$ \\ Bernardo Innocenti ${ }^{3}$ \\ Susanne Fuchs-Winkelmann ${ }^{1}$ \\ Luc Labey ${ }^{2}$
}

${ }^{1}$ Department of Orthopedics and Rheumatology, University Hospital Marburg, Germany

${ }^{2}$ European Centre for Knee Research, Smith \& Nephew, Leuven, Belgium

${ }^{3}$ BEAMS Department, Université Libre de Bruxelles, Bruxelles, Belgium

Address of Correspondence:

Thomas J. Heyse, MD

University Hospital Marburg

Department of Orthopedics and Rheumatology

Baldingerstrasse

35043 Marburg

Germany

Tel.: +49-6421-58-63 691

Fax: +49-6421-58-67 007

E-mail: heyse@med.uni-marburg.de 


\section{Abstract:}

3 Purpose:

4 It is assumed that unicondylar knee arthroplasty (UKA) features kinematics close to the

5 natural knee. Clinical studies have also shown functional benefits for UKA. There is to

6 date only little biomechanical data to support or explain these findings. The purpose of

7 this study was to investigate whether UKA is able to preserve natural knee kinematics.

8 Methods:

9 Six fresh frozen full leg cadaver specimens were prepared to be mounted in a kinematic

10 rig with six degrees of freedom for the knee joint. Three motion patterns were applied

11 before and after medial UKA: passive flexion-extension, open chain extension, and

12 squatting. During the loaded motions, quadriceps and hamstrings muscle forces were

13 applied. Infrared cameras continuously recorded the trajectories of marker frames rigidly

14 attached to femur, tibia and patella. Prior computer tomography allowed identification of

15 coordinate frames of the bones and calculations of anatomical rotations and translations.

\section{Results:}

17 Native kinematics were reproduced after UKA in all the specimens. In the unloaded knee

18 and during open chain extension, femoral rollback patterns after UKA were very close to

19 those in the native knee. During squatting, the medial femoral condyle after UKA tended

20 to be more posteriorly and superiorly with flexion and there was less tibial internal

21 rotation. The tibia was found to be in more valgus after UKA during all motion patterns.

\section{Conclusion:}

23 As ligaments, lateral compartment and patellofemoral anatomy are preserved with UKA

24 the unloaded knee closely resembles native kinematics. The slight kinematic changes that

25 were found under load are probably due to loss of the conforming medial meniscus and to

26 the mismatch in geometry and stiffness introduced by UKA. These patterns resemble

27 those found in knees with significant loss of function of the medial meniscus.

29 Keywords: Knee Kinematics, Biomechanics, Unicondylar Knee Arthroplasty, Rollback,

30 UKA




\section{Introduction:}

2 Kinematics is the description of the motion of objects (such as a femur or a tibia) without

3 considering the underlying driving forces behind it. Relative motion between two objects

4 may include translations, rotations or combinations of both.

5 The knee joint may easily be mistaken for an articulating hinge. A milestone for the

6 understanding of normal knee kinematics in relation to joint geometry was contributed by

7 the group of Pinskerova and co-workers [11,14]. The medial femoral condyle sitting on a

8 concave tibial plateau was described not to move anteroposteriorly with flexion. The

9 lateral femoral condyle tended to roll backwards with flexion on a relatively flat tibia.

10 This combination equates to internal rotation of the tibia around a medial pivot with

11 flexion [11,14]. These findings were confirmed by in vivo three-dimensional weight

12 bearing kinematics using fluoroscopy and computed tomography [16].

13 The last years have deepened the knowledge of kinematics before and after implantation

14 of total knee arthroplasty (TKA). Although TKA is usually considered an extremely

15 successful operation with excellent long-term survival of implants, there is agreement

16 that native knee kinematics cannot easily be restored

$17 \quad[19,27,28]$.

18 It is assumed that unicondylar knee arthroplasty (UKA) features kinematics closer to the

19 natural knee than TKA. Functional benefits for UKA over TKA have been described in

20 clinical studies [17,22]. However, there are only few biomechanical studies to support the

21 findings and promising results from clinical series [10].

22 Suggs et al used a robotic testing system to report on the important role of the ACL for

23 success of UKA [24]. The important role of the ACL was highlighted by another study

24 focusing on ACL reconstruction with UKA using a surgical navigation system with

25 manual stability testing over the knee [7]. Another paper reported on a comparison

26 between passive knee kinematics and stability before and after UKA and TKA, also using

27 a navigation system [5]. They showed that UKA increased varus-valgus stability in

28 extension and restored internal-external rotation of the knee during passive motion. Two

29 studies dealt with in vivo kinematics of UKA in small patient groups $[1,3]$. Some of the

30 quoted studies have described that there is significant interindividual variation in 
1 kinematics following both UKA [1,3] and TKA [28], which might be partially due to the

2 individual anatomy as well as to surgical and implant related factors.

3 Nevertheless, an efficient in vitro testing model is highly desirable to study the influence

4 of different implant types, sizes and position within the same knee as this comparative

5 data cannot easily be achieved via in vivo methods such as those mentioned above. The

6 results from fluoroscopic analyses, particularly single plane, of UKA are limited in

7 accuracy and may not be able to show subtle differences [25] that may exist between

$8 \quad$ UKA and native knee kinematics.

10 Therefore, the aim of this project is to determine how knee kinematics before and after 11 medial UKA compare using a validated kinematic rig for cadaver testing under different 12 conditions [27]. A first focus was put on the rollback pattern of the femoral condyles. A 13 second focus was put on the relative orientation of femur and tibia in terms of rotation 14 and valgus angle.

15 It was hypothesized that there would be no major differences in rollback patterns between 16 native knee and after UKA during passive motion. In the loaded knee, it was also 17 hypothesized that there could be some more anteroposterior translation between tibia and 18 femur in the medial compartment and, as a consequence, less tibial internal rotation 19 during squatting, due to a rather flat design of the polyethylene inlay of a UKA in

20 comparison with the stabilizing and centering function of an intact meniscus. Finally, it 21 was hypothesized that all kinematics changes due to UKA would be consistent among 22 specimens. 


\section{Material and Methods:}

2 Three left and three right fresh frozen human legs (mean age 74.2 \pm 15.2 years, 5 male, 1

3 female) were disarticulated at the level of the hip. All had functional ligaments and

4 showed no major deformities of the knee.

5 The experiments were performed according to a previously reported and validated

6 methodology. More detailed information on the underlying science can be retrieved from

7 this publication. For ease of understanding, the main features of the methodology are

8 described hereafter. It was shown that our technique is sufficiently accurate and precise to

9 detect differences in translations (and lengths) and rotations of less than $2 \mathrm{~mm}$ and 2

10 degrees respectively [27].

11 Three frames with four spherical infrared reflective markers each were rigidly attached to

12 the tibia, the femur, and the patella. Volumetric computed tomography scans on a sixty

13 four-row helical multidetector computed tomography (MDCT) scanner (General Electric

14 Lightspeed VCT; General Electric, Milwaukee, WI, USA) with a slice thickness of 1.25

$15 \mathrm{~mm}$ were made of the frozen specimens. For surface reconstruction and identification of

16 osseous landmark the Mimics 13.1 software and its Med-CAD Module interface

17 (Materialise, Haasrode, Leuven, Belgium) were used.

18 At the day of testing, the thawed knees were transected $32 \mathrm{~cm}$ proximal and $28 \mathrm{~cm}$ distal

19 to the knee joint. Skin and subcutaneous tissue were removed preserving the articular

20 capsule, ligaments, and tendons. Femur and tibia were embedded in holding containers

21 with polymethylmethacrylate, properly aligned in frontal and sagittal planes. The medial

22 and lateral hamstrings tendons were prepared for attachment to constant load springs (50

$23 \mathrm{~N}$ each). The quadriceps tendon was prepared to be clamped to a motor. Springs and

24 motor were fixed to the knee rig in such a way to replicate the moment arms of the

25 muscles. The knees were mounted in a kinematic rig serving as a dynamic knee simulator

26 system (Figure 1).

27 The system allows for recording motions and forces in a knee joint during squatting and

28 open chain motions. The virtual hip joint can move up and down, thus flexing and

29 extending the knee joint. A virtual ankle joint has all rotational degrees of freedom and

30 can slide medio-laterally. The flexion angle of the knee is the only degree of freedom

31 directly controlled by moving the hip vertically with constant speed. All other degrees of 
1 freedom of the knee are left free and allow motion according to individual anatomic

2 features or different testing conditions such as the presence of implants.

3 The specimens were subjected to three motion patterns: passive flexion-extension cycles,

4 an open chain extension with $3 \mathrm{~kg}$ of load hung to the distal tibia, and a squat between

$530^{\circ}$ and $120^{\circ}$ of flexion with a constant vertical ankle force of $136 \mathrm{~N}$ as an equivalent to

6 the ground reaction force (Figure 1).

7 Six MX40+ infrared cameras continuously recorded the trajectories of the markers 8 attached to the tibia, femur, and patella during all activities. The measured marker 9 trajectories were processed in Nexus (Vicon Motion Systems, Oxford, UK) using a 10 specifically designed data processing pipeline to reconstruct and label the trajectories and

11 fill any gaps in the data. Trajectories were filtered using a Woltring filtering routine and 12 the data were exported as C3D files for further processing in Matlab (R2010b, The 13 MathWorks, Natick, MA, USA).

14 Based on the CT, 3D models of tibia and femur were made and bony landmarks 15 identified to determine coordinate frames for the bones. The marker trajectories were 16 transformed to anatomical meaningful rotations and translations as proposed by Grood 17 and Suntay [9]. Tibial axial rotation, tibia position in the coronal plane (varus-valgus), 18 and translations of the femoral condyle centers in anteroposterior (AP) and mediolateral 19 (ML) direction, were obtained as a function of flexion angle (as in Figures $3-6$ ).

20 After testing the native knee, a medial UKA was performed and the same tests were 21 redone. Each specimen received an Accuris UKA system (Smith \& Nephew, Memphis,

22 TN, USA). The knees were operated using a minimal-invasive medial parapatellar 23 approach using a modular metal-backed tibia with a conventional polyethylene (PE) inlay 24 and an Oxinium femoral component. Bone cuts were made aiming for a perfectly 25 balanced joint with a $9 \mathrm{~mm}$ polyethylene (PE) inlay.

26 For each motion test, patterns and ranges of motion of internal tibial rotation, varus angle

27 as well as medial translation was compared before and after UKA. The translations of the 28 femoral condyle centers, projected on the tibial plateau and scaled to the maximum AP 29 width of the native tibial plateau, were also compared before and after UKA. After 30 alignment based on the specimen's tibial anatomical axes, these projected translations 31 were then overlaid on the axial view reconstructed from the tibial plateau of a typical 
1 specimen, with and without UKA. To allow direct comparison, all motion patterns were 2 resampled at intervals of one degree of flexion and within a common range of knee 3 flexion for all knee specimens.

4

\section{Statistical analysis}

6 Average motion patterns and standard deviations were calculated for each test. A paired 7 student's t-test was used to look for statistical significant differences at each flexion8 extension angle. Finally, to analyze and interpret the kinematic consistency between 9 specimens with and without UKA, a similar approach was applied as described earlier 10 [18] using within- and between-group coefficients of multiple correlation (CMC) [15]. 11 This is a statistical measure to quantify the similarity of time-normalized kinematic 12 waveforms. Its dimensionless aspect allows comparison of consistency across the 13 different conditions. In case of poor kinematic consistency of two different test 14 conditions for a given parameter result in between-group CMC having lower values than 15 the corresponding within-group $\mathrm{CMC}[8,18,23]$. The closer the $\mathrm{CMC}$ is to zero, the less 16 differences there are between two curves. 


\section{Results:}

2 The overall, qualitative impression of knee kinematics (tibial top views Figure 2) shows

3 physiologic femoral rollback patterns of the native knee joint with passive flexion and

4 squatting with substantial interindividual differences in rollback patterns (Figure 2).

5 These were largely reproduced after UKA.

6 This first impression was confirmed by a more quantitative analysis of translations

7 (antero-posterior and superior-inferior) and rotations (internal-external and varus-valgus).

8 The AP translation of the lateral femoral condyle centre (FLCC) was virtually equal

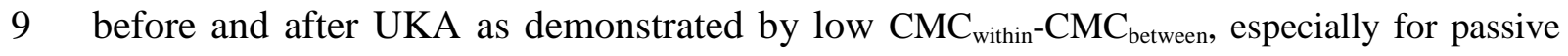

10 and squat motion (Table 1). On the medial side, the AP translation of the femoral condyle

11 centre (FMCC) after UKA matched the native knee very closely during passive and open

12 chain motion (Figure 3, Table 1). During squatting, the FMCC was positioned

13 consistently more posteriorly with UKA for all specimens and over the whole flexion

14 cycle. $\mathrm{CMC}_{\text {within-}}-\mathrm{CMC}_{\text {between }}$ clearly shows this effect for the whole curve, while statistical

15 testing on separate flexion angles did not show significance (Figure 3). The open chain

16 testing showed increased stability of the lateral compartment compared to the medial

17 compartment (Figure 3). This is also indicated by the higher $\mathrm{CMC}_{\text {within }}$ values for FLCC as

18 compared to FMCC, especially for the AP motion (Table 1). Even this somewhat atypical

19 behavior was well reproduced after UKA. The medial femoral rollback ranged from 2.4

$20 \mathrm{~mm}$ (during squatting) up to $9.6 \mathrm{~mm}$ (during passive motion) and the lateral from $2.4 \mathrm{~mm}$

21 (in open chain) up to $24 \mathrm{~mm}$ (during passive motion).

22 The first column in Table 2 documents the anteroposterior range of motion of the FMCC

23 presented as a fraction of tibial AP width (average and standard deviation for the six

24 specimens) in the three motion cycles. The data shows that the medial compartment

25 becomes increasingly more stable in AP direction under load. This is true for both the

26 native joint and after UKA. However the difference between AP excursion of FMCC

27 before and after UKA is not significant for any of the motion cycles, AP excursion of

28 FMCC systematically demonstrates the largest $\mathrm{CMC}_{\text {within }}-\mathrm{CMC}_{\text {between }}$ (Table 1). During

29 squatting FMCC moves anteriorly rather than posteriorly on average as demonstrated by

30 the largest $\mathrm{CMC}_{\text {within }}-\mathrm{CMC}_{\text {between }}$ (Table 1). 
1 Figure 4 shows that the FMCC is positioned consistently higher (approximately $3 \mathrm{~mm}$ on 2 average) after UKA for all three motion cycles. The difference is statistically significant 3 between $30^{\circ}$ and $94^{\circ}$ of flexion during passive motion, between $23^{\circ}$ and $74^{\circ}$ of flexion

4 (with some gaps) during open chain extension and between $34^{\circ}$ and $84^{\circ}$ of flexion during 5 squat. At $0^{\circ}$ flexion and around $90^{\circ}$ flexion, height of the FMCC was close to its position 6 in the native knee, showing that the knees were well balanced. On the lateral side, the 7 height of the FLCC before and after UKA remains virtually equal for all motion cycles 8 over the entire flexion range as demonstrated by systematically very low and stable 9 values for $\mathrm{CMC}_{\text {within }}-\mathrm{CMC}_{\text {between }}$ over all three motion cycles (Table 1). Differences between 10 native knee and after UKA were most significant in the coronal plane, with knee joints 11 being in less varus following surgery (Figure 6 and Table 1). No significant differences 12 were found before and after UKA in terms of mediolateral translation of the condyle 13 centres (data not shown).

15 An important focus was put on the rotation of the tibia. Internal tibial rotation showed no 16 differences before and after UKA in the passive motion (Figure 5). "During open chain, 17 between $64^{\circ}$ and $77^{\circ}$ of flexion, the native knee rotated internally when going into 18 extension from $0^{\circ}$ to $1.5^{\circ}$ (SD $11^{\circ}$ ) of internal rotation while the UKA knee remained 19 almost stable between $3.3^{\circ}$ and $3.9^{\circ}\left(\mathrm{SD} 11^{\circ}\right.$ ) of internal rotation. This difference was 20 statistically significant $(\mathrm{p}<0.05)$.

21 In squatting, there was a tendency for less internal rotation of the tibia after UKA. CMC 22 analysis outcome showed that there was less internal rotation of the tibia following UKA. 


\section{Discussion:}

2 The most important finding of this in vitro biomechanical study was that knee kinematics

3 after UKA are indeed close to those of a native knee especially under passive motion.

4 There were however some small but nevertheless significant differences, particularly in

5 terms of inferior-superior position of the medial femoral condyle and varus-valgus tilting

6 of the tibia. There was also less internal tibial rotation and a more posterior position of

7 the femoral medial condyle during squatting. The kinematic findings were highly

8 consistent over the tested specimens.

10 Based on the measurements made, the bony geometry of a native medial femoral condyle

11 and tibial plateau seems to be functionally restored by the implant. Due to difficulties in

12 reproducing an accurate contact point with UKA, the PE inlay of most UKA is however

13 relatively flat, differing from the more conforming wedge shape of the medial meniscus.

14 The importance of an intact medial meniscus for knee kinematics has recently been

15 described in a couple of biomechanical studies [2,20].

16 Allaire et al described kinematics in cadaver knees at four knee-flexion angles with a

17 (repaired) posterior root tear of the medial meniscus and after total medial meniscectomy.

18 In their study, root tears and especially a total meniscectomy led to less internal tibial

19 rotation with flexion and a little more varus. They also described a more posterior

20 position of the medial femoral condyle after total meniscectomy. Biomechanics were

21 described to return to normal after surgical repair of posterior root tears [2]. Most of those

22 kinematic effects in a knee with significant damage to the medial meniscus mirror the

23 findings made after UKA. Loss of the stabilizing and conforming function of the medial

24 meniscus results in less internal tibial rotation and more posterior position of the medial

25 femoral condyle with flexion especially under loaded conditions. This becomes evident

26 when high forces are transmitted over the knee joint as in a squat. Under less straining

27 activities such as passive motion of the knee, the remaining preserved structures of the

28 knee provide sufficient stability to maintain normal kinematics after UKA.

29 However, the tests also revealed some other (statistically significant) differences between

30 native and UKA kinematics which cannot be explained solely by the absence of the

31 medial meniscus and loss of conformity of the medial compartment. The femoral medial 
1 condyle center of the knees with UKA was positioned more superiorly and, probably as a

2 consequence of this, the tibia was more in valgus. There are two plausible reasons for this

3 result, one geometrical the other biomechanical.

4 The curvature of the femoral component ( $\mathrm{R}^{\prime}$ in Figure $7 \mathrm{a}$ ) might be slightly smaller than

5 the curvature of the native femoral condyle ( $\mathrm{R}$ in Figure $7 \mathrm{a}$ ) for example. Because the

6 UKA is balanced at $0^{\circ}$ and $90^{\circ}$, the femoral component will be flush with the native

7 articular surface at those flexion angles, but it will protrude at all intermediate flexion

8 angles as shown in Figure 7. This effect will lead to a more superior position of FMCC,

9 in any motion cycle whether loaded or unloaded. This is also what was found during the

10 tests. On the other hand, this explanation requires that femoral component curvature is

11 consistently smaller than the native condyle curvature for the six specimens, which seems

12 improbable. One would rather expect the component curvature to be smaller in some

13 specimens and larger in others.

14 Another important parameter is the mismatch in stiffness which is introduced by UKA

15 between the native articular surface (cartilage 5 to $12 \mathrm{MPa}$ ) on the lateral side versus a

16 UHMWPE insert (850 MPa) on an Oxinium femoral component (97 GPa,[12] medially.

17 When the knee is loaded, the medial compartment deforms less after UKA than in the

18 native joint (as illustrated in Table 1 by decreasing pre- vs. post-UKA consistency in

19 terms of the IS motion of FMCC with increased loading, i.e. when going from passive, over open

20 chain to squat motions). This has three consequences. First of all, in loaded conditions,

21 after UKA, the femur will be more superior than in the native knee. Secondly, due to

22 uneven compression on medial and lateral side, the tibia will tilt towards a more valgus

23 position. And finally, the PCL remains more taught than in the loaded native knee and

24 therefore the femoral medial condyle center will be held more posteriorly (Figure 7b).

25 The results presented here provide added value compared to previous experimental work

26 and gait analysis on UKA [6,13,21]. A cadaver study was also performed by Patil et al.

27 They limited their kinematics analysis and interpretation mainly to the tibial horizontal

28 plane and did not simulate hamstring forces in their setup, with a more limited amount of

29 loading conditions and simulated motion patterns. The implants used were fixed bearing

30 UKA in both studies with relatively flat PE inlays but from different manufacturers. The

31 sensitivity of the setup described in their publication seemed to be relatively low as it 
1 surprisingly failed to show differences in kinematics before and after ACL resection with

2 UKA [21] unlike other reports underlining the importance of the ACL for normal knee

3 kinematics with or without UKA [7,19,24,28]. The statement that native knee kinematics

4 and those found after UKA are identical [21] seems to simplify the complex conditions

5 following UKA surgery. The more differentiated approach used in our study, by

6 considering different motion patterns and loading regimens of daily living may therefore

7 more adequately reveal the subtle differences in knee kinematics between native knees 8 and UKA.

9 Suggs et al. used a robotic system to move the knee while applying constant forces on

10 quadriceps and hamstrings. They found no significant effect of UKA on AP position of

11 the medial condyle. UKA mainly led to a clearly more valgus orientation at $0^{\circ}$ and $30^{\circ}$ of

12 flexion, but not in deeper flexion and it also put the tibia in more external rotation over

13 most of the flexion range. Overall, these findings are similar to ours, although the details

14 of where the differences are statistically significant may differ. The authors also made the

15 link between differences in tibial axial rotation and the loss of the medial meniscus after

16 UKA, but they did not attempt to explain the differences.

17 Becker et al reported that there was no difference in stability between the native and

18 UKA knees in AP translation and rotation in all knee flexion angles independent of fixed

19 and mobile bearings in their robotic cadaver setup [4], which corresponds well with our

20 findings.

22 There are limitations to this study. Firstly, access to cadaver specimens is obviously 23 limited and only a few specimens were used. Secondly, testing conditions often include a

24 large number of tests without applying a statistical correction for multiple testing. It was

25 felt that this is however ethically justified. Thirdly, test results may be influenced by the

26 quality of specimen affected by donor factors such as age and underlying diseases as well

27 as timing of storage and treatment during testing and preparation. Finally, the testing

28 conditions could only include a limited range of activities and motions over the knee and

29 will never reflect the full spectrum of living function. To date, the interaction between

30 flexors and extensors over the knee is not fully understood nor described. Victor et al

31 described that loading the knee with hamstrings and quadriceps reduces rotation and 
1 translation compared to the passive condition. Lateral hamstring action was described to

2 be more influential on knee kinematics than medial hamstrings action [26]. In our setup

3 we used muscle forces as described in their work as it may approximate physiologic loads

4 on these muscle groups closely. We did not, however, investigate the effect of different

5 hamstring and quadriceps load combinations. We would guess that after UKA the effect

6 of presence of absence of medial hamstring load might become more visible because of

7 the loss of AP stability in the medial compartment.

8

9 It should still be acknowledged that the kinematics after UKA is indeed very close to the 10 native knee. Even for those degrees of freedom where we found statistically significant 11 differences, those differences were small enough to probably go unnoticed by the patient 12 during his daily activities. Indeed, axial rotation discrepancies before and after UKA were 13 at most $3.5^{\circ}$ and the difference in varus after UKA was limited to a maximal $3.1^{\circ}$. 


\section{Conclusion:}

2 The data presented in this article explain that UKA seems to resemble kinematics of a 3 native knee closely with some features of knees with a resected medial meniscus under 4 loaded conditions.

5 It was shown that the medial femoral condyle center is consistently positioned more 6 superiorly with respect to the native knee, both in unloaded and loaded conditions. 7 Despite these findings, kinematics after UKA were overall very close to the native knee. 


\section{Acknowledgements:}

2 The authors wish to thank all donors, who dedicated their mortal remains to science.

3 Research, such as in this project, to deepen the understanding about function of the

4 human body would be impossible without such act of selflessness.

5 Rest blinded for review process. 


\section{References:}

1. Akizuki S, Mueller JK, Horiuchi H, Matsunaga D, Shibakawa A, Komistek RD (2009) In vivo determination of kinematics for subjects having a Zimmer Unicompartmental High Flex Knee System. J Arthroplasty 24 (6):963-971

2. Allaire R, Muriuki M, Gilbertson L, Harner CD (2008) Biomechanical consequences of a tear of the posterior root of the medial meniscus. Similar to total meniscectomy. J Bone Joint Surg Am 90 (9):1922-1931

3. Argenson JN, Komistek RD, Aubaniac JM, Dennis DA, Northcut EJ, Anderson DT, Agostini S (2002) In vivo determination of knee kinematics for subjects implanted with a unicompartmental arthroplasty. J Arthroplasty 17 (8):1049-1054

4. Becker R, Mauer C, Starke C, Brosz M, Zantop T, Lohmann CH, Schulze M (2012) Anteroposterior and rotational stability in fixed and mobile bearing unicondylar knee arthroplasty: a cadaveric study using the robotic force sensor system. Knee Surg Sports Traumatol Arthrosc [Epub ahead of print]

5. Casino D, Martelli S, Zaffagnini S, Lopomo N, Iacono F, Bignozzi S, Visani A, Marcacci M (2009) Knee stability before and after total and unicondylar knee replacement: in vivo kinematic evaluation utilizing navigation. J Orthop Res 27 (2):202-207.

6. Catani F, Benedetti MG, Bianchi L, Marchionni V, Giannini S, Leardini A (2012) Muscle activity around the knee and gait performance in unicompartmental knee arthroplasty patients: a comparative study on fixed- and mobile-bearing designs. Knee Surg Sports Traumatol Arthrosc 20 (6):1042-1048.

7. Citak M, Bosscher MR, Citak M, Musahl V, Pearle AD, Suero EM Anterior cruciate ligament reconstruction after unicompartmental knee arthroplasty. Knee Surg Sports Traumatol Arthrosc 19 (10):1683-1688

8. Fitoussi F, Maurel N, Diop A, Laassel EM, Ilharreborde B, Presedo A, Mazda K, Pennecot GF (2009) Upper extremity kinematics analysis in obstetrical brachial plexus palsy. Orthop Traumatol Surg Res 95 (5):336-342.

9. Grood ES, Suntay WJ (1983) A joint coordinate system for the clinical description of three-dimensional motions: application to the knee. J Biomech Eng 105 (2):136144

10. Heyse TJ, Khefacha A, Peersman G, Cartier P (2012) Survivorship of UKA in the middle-aged. The Knee 19 (5):585-591.

11. Hill PF, Vedi V, Williams A, Iwaki H, Pinskerova V, Freeman MA (2000) Tibiofemoral movement 2: the loaded and unloaded living knee studied by MRI. J Bone Joint Surg Br 82 (8):1196-1198

12. Hobbs LW, Benezra Rosen V, Mangin SP, Treska M, Hunter G ( 2005) Oxidation microstructures and interfaces in the oxidized zirconium knee. Int $\mathbf{J}$ Appl Ceram Technol 2(3):221-246

13. Hopkins AR, New AM, Rodriguez-y-Baena F, Taylor M (2010) Finite element analysis of unicompartmental knee arthroplasty. Med Eng Phys 32 (1):14-21.

14. Iwaki H, Pinskerova V, Freeman MA (2000) Tibiofemoral movement 1: the shapes and relative movements of the femur and tibia in the unloaded cadaver knee. $\mathbf{J}$ Bone Joint Surg Br 82 (8):1189-1195 
15. Kadaba MP, Ramakrishnan HK, Wootten ME, Gainey J, Gorton G, Cochran GV (1989) Repeatability of kinematic, kinetic, and electromyographic data in normal adult gait. J Orthop Res 7 (6):849-860.

16. Komistek RD, Dennis DA, Mahfouz M (2003) In vivo fluoroscopic analysis of the normal human knee. Clin Orthop Relat Res 410:69-81

17. Laurencin CT, Zelicof SB, Scott RD, Ewald FC (1991) Unicompartmental versus total knee arthroplasty in the same patient. A comparative study. Clin Orthop Relat Res 273:151-156

18. Leffler J, Scheys L, Plante-Bordeneuve T, Callewaert B, Labey L, Bellemans J, Franz A (2012) Joint kinematics following bi-compartmental knee replacement during daily life motor tasks. Gait Posture 36 (3):454-460.

19. Mahfouz MR, Komistek RD, Dennis DA, Hoff WA (2004) In vivo assessment of the kinematics in normal and anterior cruciate ligament-deficient knees. J Bone Joint Surg Am 86-A Suppl 2:56-61

20. Muriuki MG, Tuason DA, Tucker BG, Harner CD Changes in tibiofemoral contact mechanics following radial split and vertical tears of the medial meniscus an in vitro investigation of the efficacy of arthroscopic repair. J Bone Joint Surg Am 93 (12):1089-1095

21. Patil S, Colwell CW, Jr., Ezzet KA, D'Lima DD (2005) Can normal knee kinematics be restored with unicompartmental knee replacement? J Bone Joint Surg Am 87 (2):332-338

22. Rougraff BT, Heck DA, Gibson AE (1991) A comparison of tricompartmental and unicompartmental arthroplasty for the treatment of gonarthrosis. Clin Orthop Relat Res 273:157-164

23. Serrao M, Spaich EG, Andersen OK (2012) Modulating effects of bodyweight unloading on the lower limb nociceptive withdrawal reflex during symmetrical stance. Clin Neurophysiol 123 (5):1035-1043.

24. Suggs JF, Li G, Park SE, Sultan PG, Rubash HE, Freiberg AA (2006) Knee biomechanics after UKA and its relation to the ACL--a robotic investigation. $J$ Orthop Res 24 (4):588-594

25. Van Duren BH, Pandit H, Beard DJ, Murray DW, Gill HS (2009) Accuracy evaluation of fluoroscopy-based $2 \mathrm{D}$ and $3 \mathrm{D}$ pose reconstruction with unicompartmental knee arthroplasty. Med Eng Phys 31 (3):356-363

26. Victor J, Labey L, Wong P, Innocenti B, Bellemans J (2010) The influence of muscle load on tibiofemoral knee kinematics. J Orthop Res 28 (4):419-428.

27. Victor J, Van Glabbeek F, Vander Sloten J, Parizel PM, Somville J, Bellemans J (2009) An experimental model for kinematic analysis of the knee. J Bone Joint Surg Am 91 Suppl 6:150-163

28. Yoshiya S, Matsui N, Komistek RD, Dennis DA, Mahfouz M, Kurosaka M (2005) In vivo kinematic comparison of posterior cruciate-retaining and posterior stabilized total knee arthroplasties under passive and weight-bearing conditions. J Arthroplasty 20 (6):777-783

43

44

45 


\section{Captions to Figures:}

\section{Figure 1:}

4 Setup photographs of open chain testing (left) and a squat (right): R: Rig; F: Femur; T:

5 Tibia; *: frames with infrared reflective markers; H: hamstrings with load springs; Q:

6 clamped quadriceps tendon.

\section{Figure 2:}

9 The rollback behavior before and after UKA for the tested motions for all specimens.

10 Rollback is displayed from the projected displacement of the femoral condyle centers,

11 overlaid on the tibial plateau, and calculated in relation to the tibial plateau AP size of the 12 corresponding specimen. Tibial images with and without UKA are generic depictions 13 created from a native knee and only serve as illustrations. In these plots, the medial and 14 lateral femoral condyles centers (dots) are linked by a line whose color reflects the 15 corresponding flexion angle, thus the progressive color gradient from full extension 16 (darker color) to full flexion (lighter color).

\section{Figure 3:}

19 Translations of the Medial femoral condyle centers (FMCC) are expressed as relative to

20 the AP size of the tibial plateau. Values for each knee were calculated using the 21 respective specimen's dimensions, then averaged in the following graphs. Standard 22 deviations are shown with shaded areas of corresponding color. Regions of non23 significant differences are shown with faded out colors.

\section{Figure 4:}

26 Average inferior-superior translation between femur and tibia as a function of flexion

27 before and after UKA for the three motion tests. Standard deviations are shown with 28 shaded areas of corresponding color. Regions of non-significant differences are shown 29 with faded out colors. 


\section{Figure 5:}

2 Average internal rotation of the tibia as a function of flexion before and after UKA for

3 the three motion tests: Standard deviations are shown with shaded areas of corresponding

4 color. Regions of non-significant differences are shown with faded out colors.

6 Figure 6:

7 Average varus angle between femur and tibia as a function of flexion before and after

8 UKA for the three motion tests. Standard deviations are shown with shaded areas of 9 corresponding color. Regions of non-significant differences are shown with faded out 10 colors.

\section{Figure 7:}

13 Schematic view of the effect of geometrical (a) and stiffness (b) mismatch between the 14 native and the replaced medial compartment on height and AP position of the FMCC.

18 Table 1: Overview of within-group CMC, between-group CMC values and the absolute

19 difference between both for all analysed components of pre- and post-UKA knee 20 kinematics.

22 Table 2: Average, minimum and maximum values for AP excursion of FMCC, varus 23 tibial tilting excursion and internal tibial rotation excursion. Significant differences $24 \quad(\mathrm{p}<0.05)$ are indicated with an asterisk. 
Click here to download high resolution image
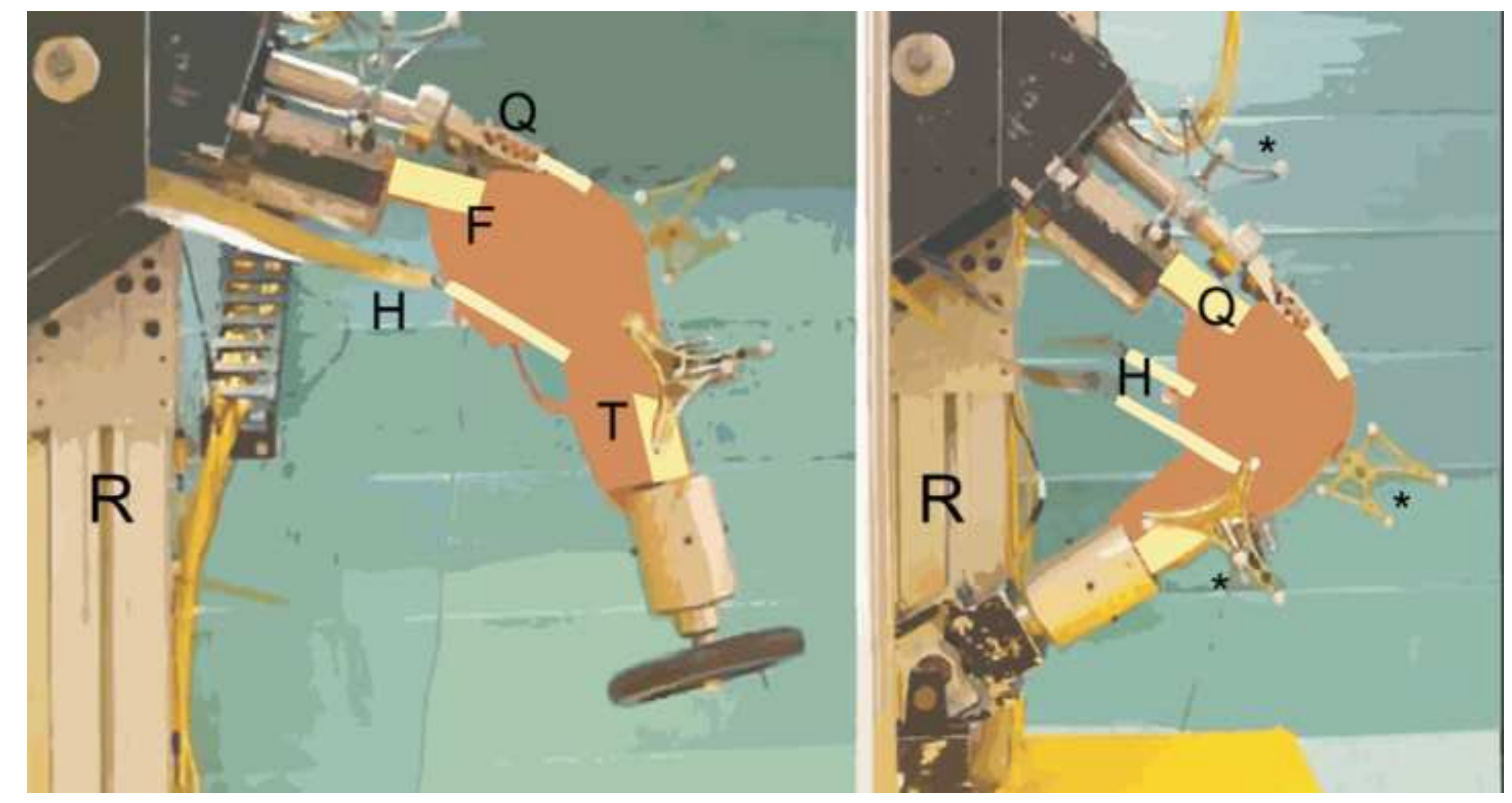
Click here to download high resolution image

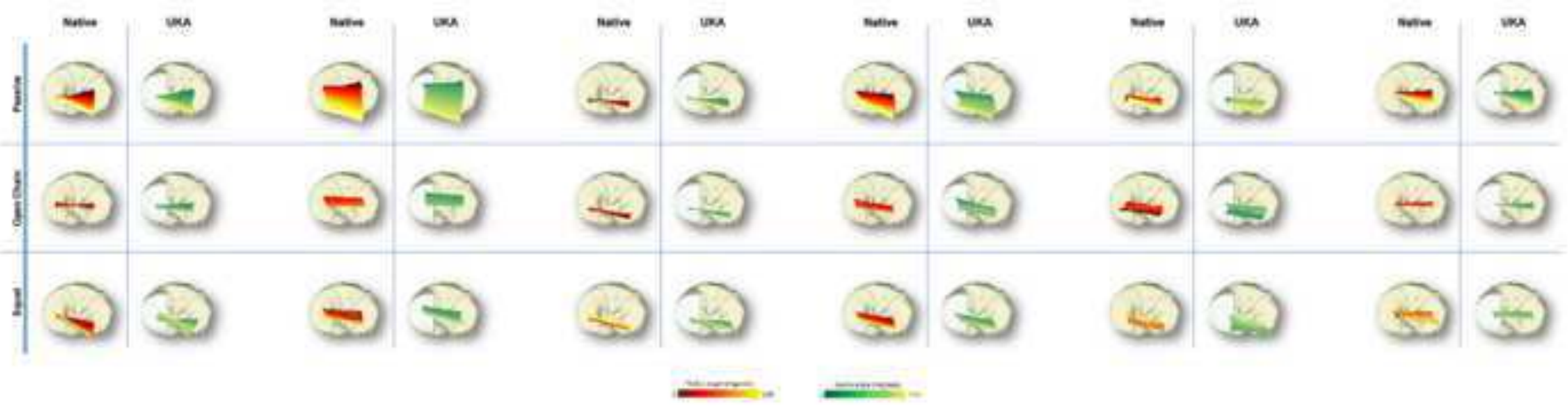



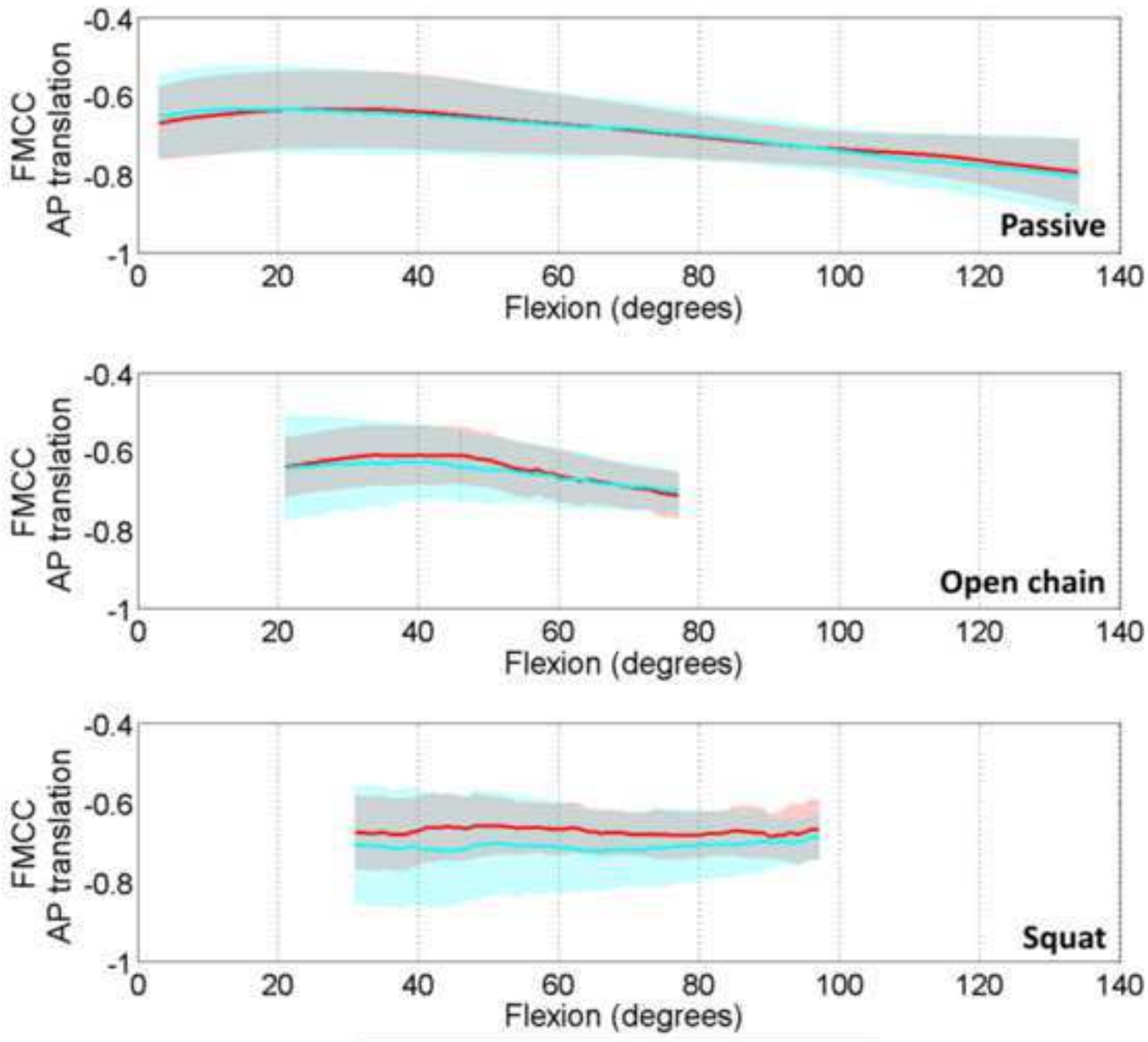

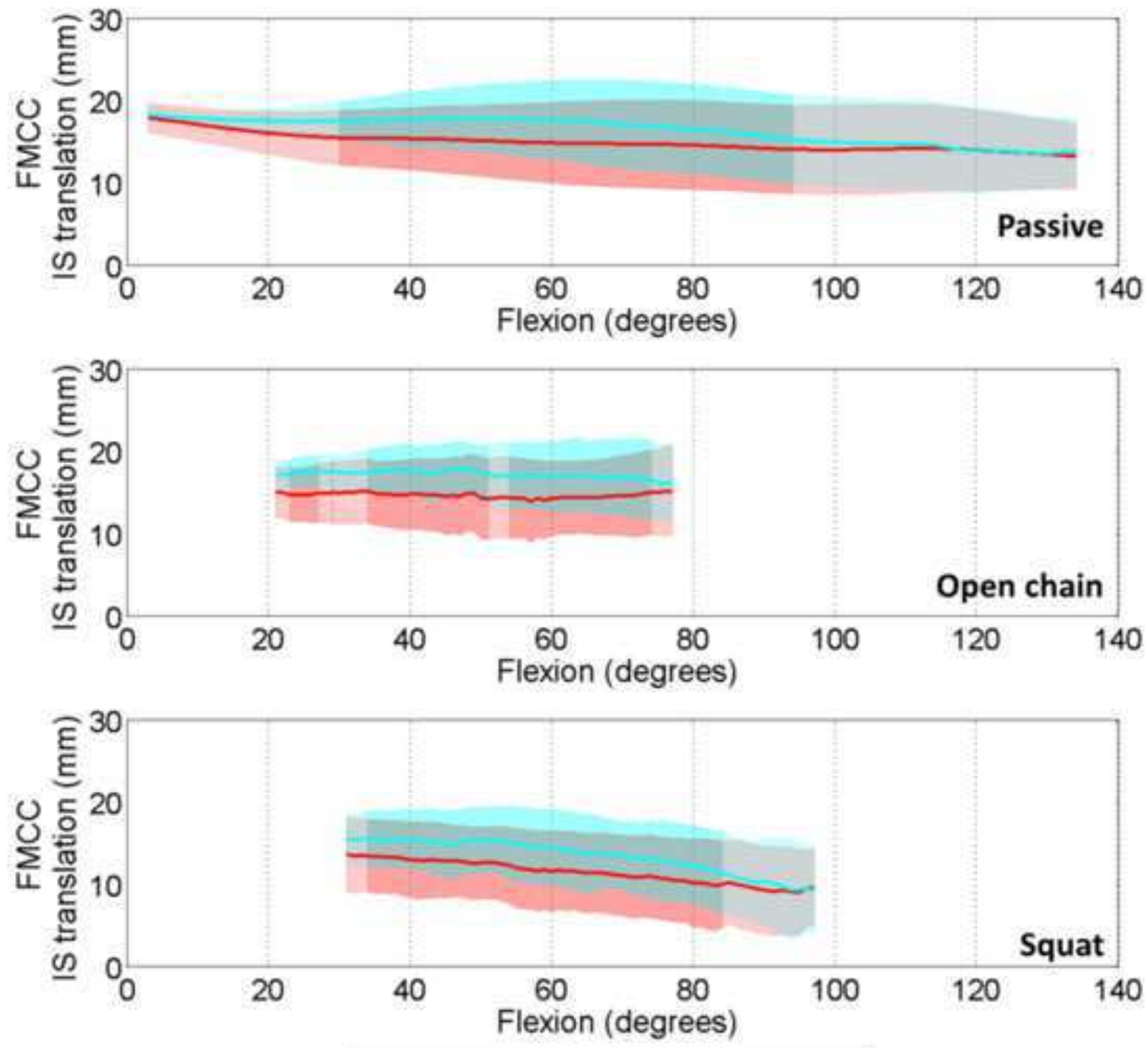

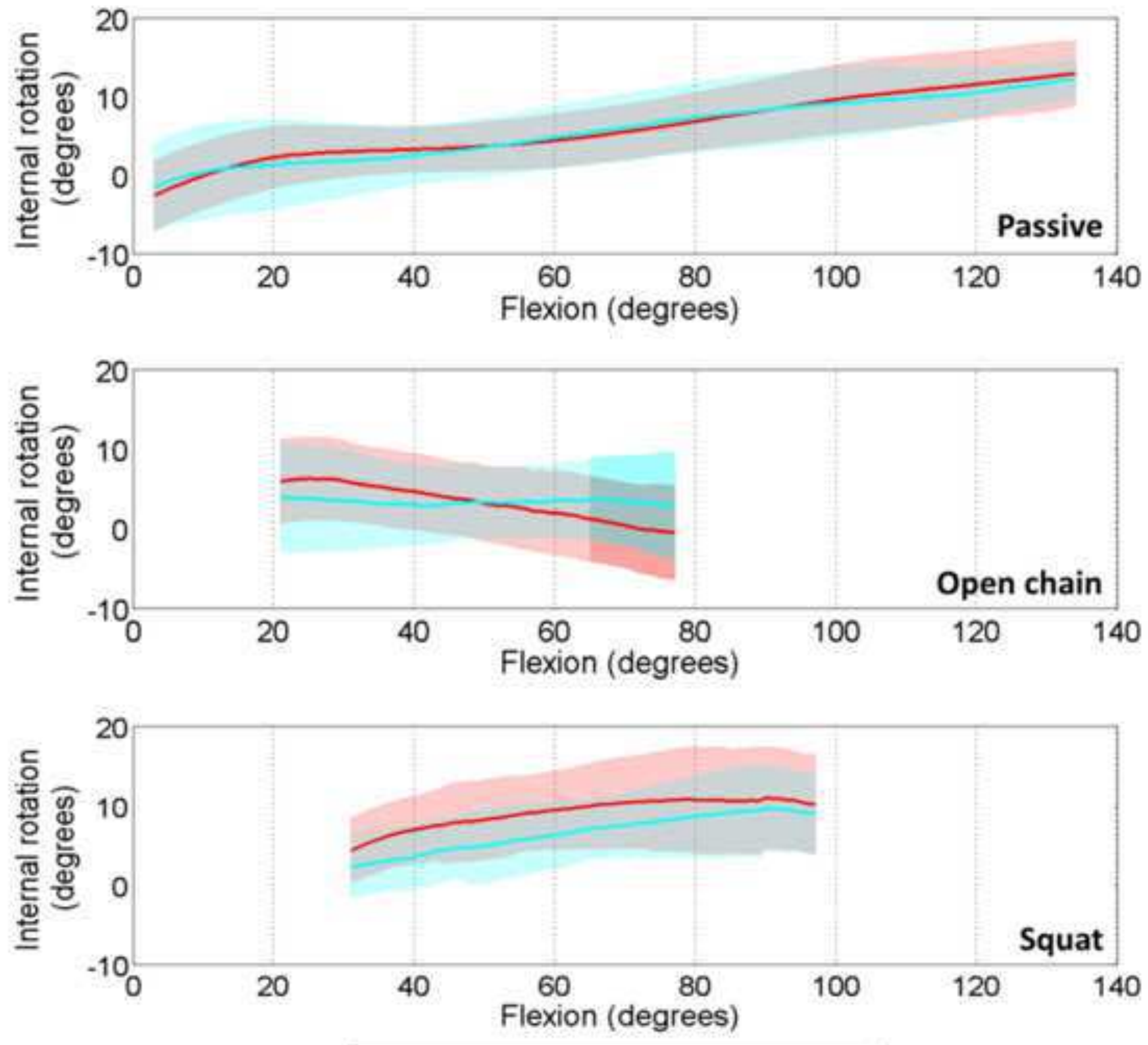

Native 

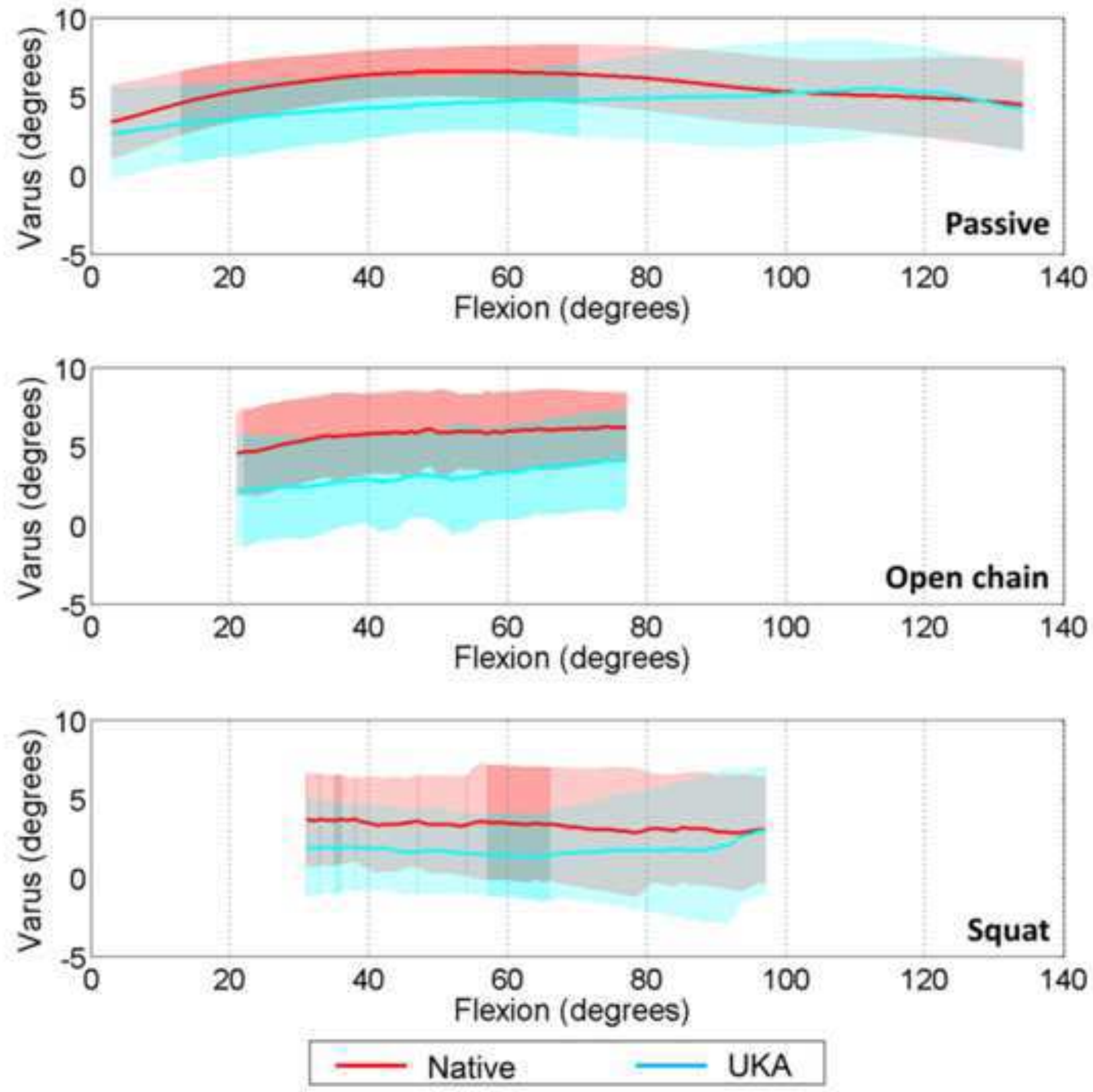
(a)

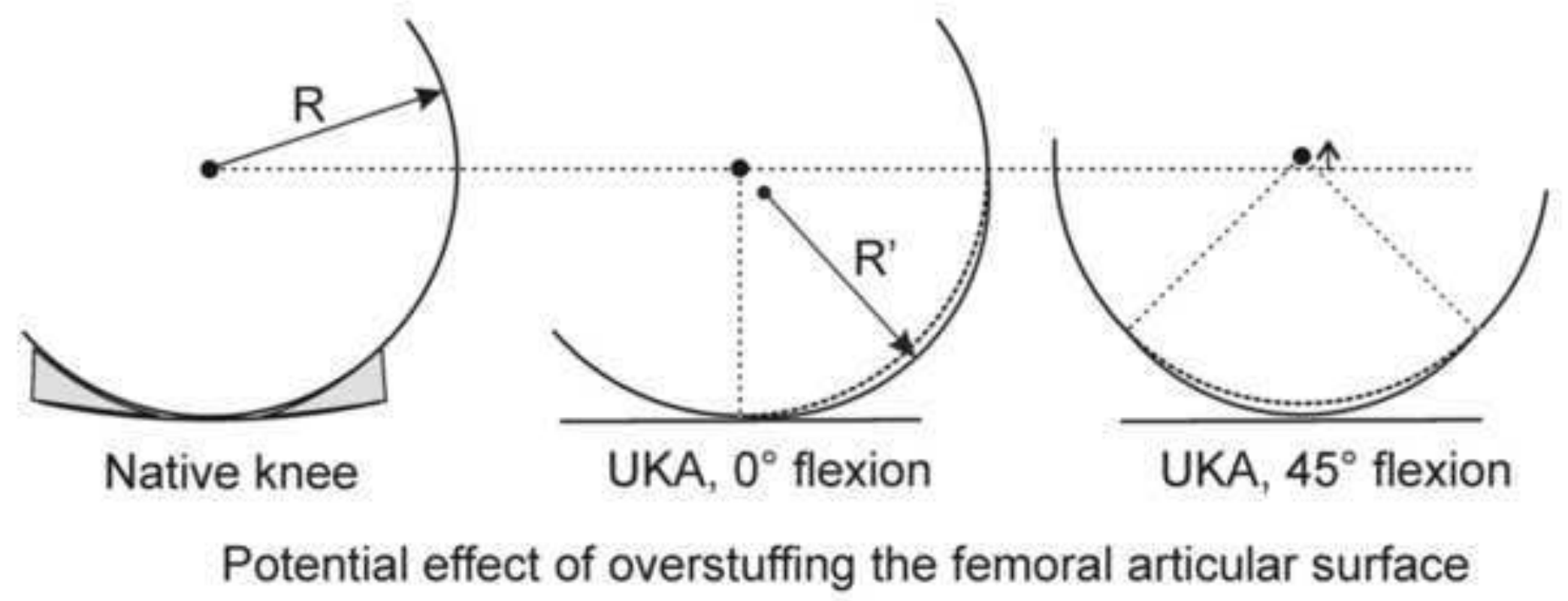

Native, unloaded

Native, loaded

UKA, loaded

(b)
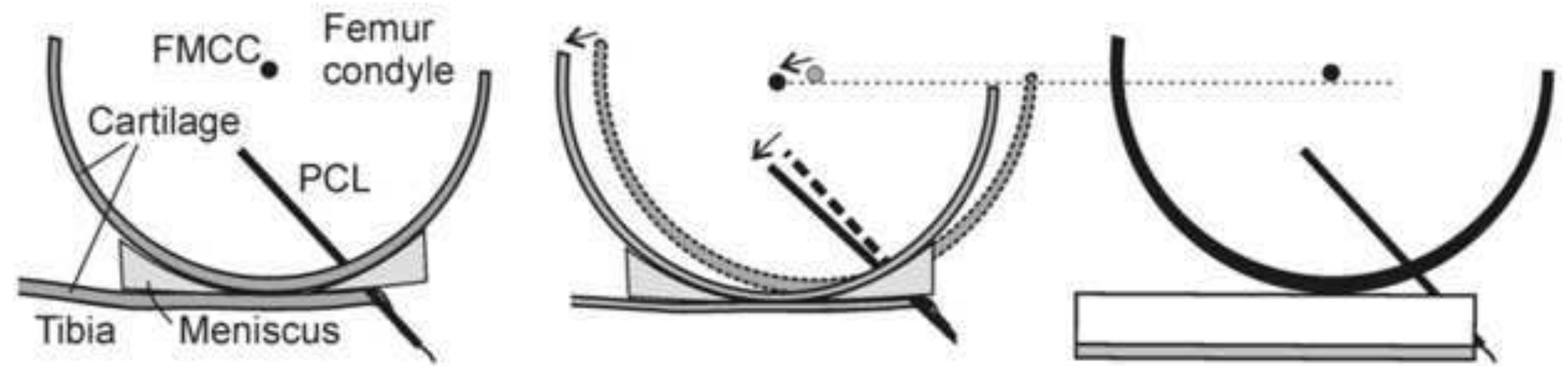

Anterior and inferior femoral shift as a consequence of load 
Table 1:

\begin{tabular}{|l|l|c|c|c|}
\hline & & $\mathbf{C M C}_{\text {within }}$ & $\mathbf{C M C}_{\text {between }}$ & $\mathbf{C M C}_{\text {within }} \mathbf{C M C}_{\text {between }}$ \\
\hline \multirow{4}{*}{ Passive } & Varus/valgus & 0.711 & 0.697 & 0.014 \\
\cline { 2 - 5 } & Tibial rotation & 0.593 & 0.579 & 0.015 \\
\cline { 2 - 5 } & AP motion of FLCC & 0.409 & 0.402 & 0.007 \\
\cline { 2 - 5 } & IS motion of FLCC & 0.818 & 0.809 & 0.009 \\
\cline { 2 - 5 } & AP motion of FMCC & 0.535 & 0.497 & 0.038 \\
\cline { 2 - 5 } & IS motion of FMCC & 0.841 & 0.829 & 0.013 \\
\hline \multirow{5}{*}{ Open Chain } & Varus/valgus & 0.928 & 0.858 & 0.070 \\
\cline { 2 - 5 } & Tibial rotation & 0.903 & 0.890 & 0.014 \\
\cline { 2 - 5 } & AP motion of FLCC & 0.953 & 0.898 & 0.055 \\
\cline { 2 - 5 } & IS motion of FLCC & 0.976 & 0.971 & 0.006 \\
\cline { 2 - 5 } & AP motion of FMCC & 0.892 & 0.850 & 0.042 \\
\cline { 2 - 5 } & IS motion of FMCC & 0.963 & 0.926 & 0.037 \\
\hline \multirow{5}{*}{ Squat } & Varus/valgus & 0.887 & 0.865 & 0.022 \\
\cline { 2 - 5 } & Tibial rotation & 0.862 & 0.803 & 0.059 \\
\cline { 2 - 5 } & AP motion of FLCC & 0.818 & 0.809 & 0.009 \\
\cline { 2 - 5 } & IS motion of FLCC & 0.876 & 0.862 & 0.014 \\
\cline { 2 - 5 } & AP motion of FMCC & 0.846 & 0.758 & 0.088 \\
\cline { 2 - 5 } & IS motion of FMCC & 0.858 & 0.805 & 0.053 \\
\hline
\end{tabular}


Table 2:

\begin{tabular}{|c|l|l|l|l|}
\hline & & $\begin{array}{l}\text { AP motion of } \\
\text { FMCC }\end{array}$ & Varus tilt & Internal rotation \\
\hline \multirow{2}{*}{ Passive } & Native & $-0.16(-0.46-0.04)$ & $3.2^{\circ}\left(-7.3^{\circ}-8.1^{\circ}\right)$ & $16^{\circ}\left(9.6^{\circ}-24.3^{\circ}\right)$ \\
\cline { 2 - 5 } & UKA & $-0.16(-0.45--0.06)$ & $3.0^{\circ}\left(-5.1^{\circ}-12.4^{\circ}\right)$ & $13^{\circ}\left(9^{\circ}-19^{\circ}\right)$ \\
\hline \multirow{2}{*}{$\begin{array}{c}\text { Open } \\
\text { Chain }\end{array}$} & Native & $-0.11(-0.16--0.06)$ & $0.8^{\circ}\left(-2.4^{\circ}-4.2^{\circ}\right)$ & $-6.6^{\circ}\left(-14.7^{\circ}--1.1^{\circ}\right)^{*}$ \\
\cline { 2 - 5 } Squat & UKA & $-0.07(-0.18--0.01)$ & $1.8^{\circ}\left(-3.8^{\circ}-6.1^{\circ}\right)$ & $-1^{\circ}\left(-12.1^{\circ}-6.2^{\circ}\right)^{*}$ \\
\hline \multirow{2}{*}{ Native } & $-0.04(-0.15-0.16)$ & $-1.7^{\circ}\left(-5.2^{\circ}-4.5^{\circ}\right)$ & $6.7^{\circ}\left(4^{\circ}-16^{\circ}\right)$ \\
\cline { 2 - 5 } & UKA & $0.04(-0.14-0.25)$ & $1.6^{\circ}\left(-6.8^{\circ}-8^{\circ}\right)$ & $7.9^{\circ}\left(3.9^{\circ}-19.9^{\circ}\right)$ \\
\hline
\end{tabular}

